\title{
A Multidisciplinary Toxicity Team for Cancer Immunotherapy-Related Adverse Events
}

\author{
Jarushka Naidoo, MBBCha; Jiajia Zhang, MD, PhDa; Evan J. Lipson, MDa; Patrick M. Forde, MBBCha; Karthik Suresh, MD; \\ Kendall F. Moseley, MDc; Seema Mehta, MDd; Shawn G. Kwatra, MDe; Alyssa M. Parian, MDf; Amy K. Kim, MD;; \\ John C. Probasco, MD, PhDg; Rosanne Rouf, MD ; Jennifer E. Thorne, MD, PhDij; Satish Shanbhag, MDa; \\ Joanne Riemer, RN, BSN; Ami A. Shah, MDk; Drew M. Pardoll, MD, PhDa; Clifton O. Bingham III, MD*; \\ Julie R. Brahmer, MD, MHSa; and Laura C. Cappelli, MD, MHS
}

\section{ABSTRACT}

Background: Immune checkpoint inhibitors (ICls) may cause immunerelated adverse events (irAEs). Methods to obtain real-time multidisciplinary input for irAEs that require subspecialist care are unknown. This study aimed to determine whether a virtual multidisciplinary immunerelated toxicity (IR-tox) team of oncology and medicine subspecialists would be feasible to implement, be used by oncology providers, and identify patients for whom multidisciplinary input is sought. Patients and Methods: Patients treated with ICls and referred to the IR-tox team in August 2017 through March 2018 were identified. Feasibility was defined as receipt of electronic referrals and provision of recommendations within 24 hours of referral. Use was defined as the proportion of referring providers who used the team's recommendations, which was determined through a postpilot survey. Demographics and tumor, treatment, and referral data were collected. Patient features and irAE associations were analyzed. Results: The IR-tox team was found to be feasible and used: 117 referrals from 102 patients were received in 8 months, all providers received recommendations within 24 hours, $100 \%$ of surveyed providers used the recommendations, and $74 \%$ changed patient management based on IR-tox team recommendations. Referrals were for suspected irAEs $(n=106$; $91 \%)$ and suitability to treat with $\mathrm{ICls}(\mathrm{n}=11 ; 10 \%)$. In referred patients, median age was 64 years, 54\% were men, $13 \%$ had prior autoimmunity, and $46 \%$ received $\mathrm{ICl}$ combinations versus monotherapy (54\%). The most commonly referred toxicities were pneumonitis (23\%), arthritis (16\%), and dermatitis (15\%); $15 \%$ of patients had multisystem toxicities. Multiple referrals were more common in those treated with combination $\mathrm{ICls}$ (odds ratio $[\mathrm{OR}], 6.0 ; P=.035$ ) or with multisystem toxicities (OR, $8.1 ; P=.005)$. The IR-tox team provided a new multidisciplinary forum to assist providers in diagnosing and managing complex irAEs. This model identifies educational and service needs, and patients with irAEs for whom multidisciplinary care is most sought. Conclusions: A virtual multidisciplinary toxicity team for irAEs was a feasible and used service, and facilitated toxicity identification and management.

J Natl Compr Canc Netw 2019;17(6):712-720 doi: 10.6004/jnccn.2018.7268

See JNCCN.org for supplemental online content.

\section{Background}

Multidisciplinary tumor boards are a mechanism in which oncologists and subspecialists discuss challenging cases to optimize patient care. Prospective studies of these forums have demonstrated that multidisciplinary input can alter diagnosis and positively influence management across tumor types..$^{1-5}$ More recently, specialized molecular tumor boards that interpret patient genomic data to guide clinical care have gained popularity. ${ }^{6}$

In the field of cancer immunotherapy, emerging immune-related toxicities require multidisciplinary management. Due to their mechanism of action, immune checkpoint inhibitors (ICIs) can cause patients to develop a variety of organ-specific immune-related adverse events (irAEs). ${ }^{7}$ Similarly, patients may have other processes, such as infection or progressive cancer, that mimic irAEs. Early evaluation and diagnosis is critical and associates with successful irAE outcomes. ${ }^{8,9}$ Diagnosis and management of selected irAEs may be challenging and often requires the input of at least one non-oncology specialist for specific physical examination, interpretation of laboratory tests or imaging, completion of a diagnostic procedure, or guidance on immunosuppression for steroid-refractory irAEs. Additionally, high-risk patients with preexisting autoimmune diseases, prior organ/ bone marrow transplants, or recalcitrant irAEs may necessitate input from multiple subspecialists. Oncology physicians and nurses are central to this consultation, to

aBloomberg-Kimmel Institute for Cancer Immunotherapy, Sidney Kimmel Comprehensive Cancer Center at Johns Hopkins; 'b Department of Medicine, Division of Pulmonary and Critical Care Medicine, 'Department of

Medicine, Division of Endocrinology, Diabetes \& Metabolism, ${ }^{\mathrm{d}}$ Department

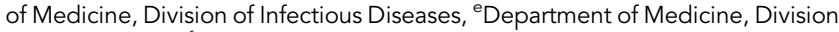
of Dermatology, fDepartment of Medicine, Division of Gastroenterology,

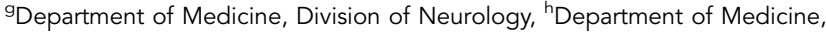
Division of Cardiology, 'Department of Medicine, Division of Ophthalmology, 'Department of Epidemiology, and kDepartment of Medicine, Division of Rheumatology, Johns Hopkins University School of Medicine, Baltimore, Maryland. 
address ICI discontinuation or suitability for future immunotherapy. Most literature regarding irAEs recommends a multidisciplinary approach but lacks practical guidance on how this can be achieved in real time. Important considerations include lack of familiarity with certain irAEs, that irAEs may occur at unpredictable times when specialists are not available, and the need for expeditious discussions to ensure medicine and oncology recommendations are not conflicting.

This study aimed to formalize the multidisciplinary collaborations between oncology and medicine subspecialists for irAE management. Our goal was to recruit oncology and medical subspecialists with expertise in irAE diagnosis and management to provide real-time input on irAEs via an electronic platform, and to test the feasibility and use of this immune-related toxicity (IR-tox) team.

\section{Patients and Methods}

\section{IR-Tox Team Operations}

The IR-tox team was proposed as a collaboration between medical oncology (J.N., Co-Chair) and medicine subspecialists (L.C.C., Co-Chair). This multidisciplinary group consisted of subspecialists with expertise in irAE management, including medical oncologists $(n=8)$, oncology nurses $(n=4)$, and subspecialists from rheumatology $(n=4)$, pulmonology $(n=3)$, gastroenterology $(n=3)$, neurology $(n=2)$, endocrinology $(n=2)$, dermatology $(n=2)$, ophthalmology $(n=2)$, cardiology $(n=1)$, infectious diseases $(n=1)$, and hematology $(n=1)$. The co-chairs proposed a pilot program for oncology providers (faculty, fellows, nurses) who administer ICIs to patients in a tertiary cancer center to electronically consult the IR-tox team for assistance in the diagnosis and management of suspected irAEs or related issues. An electronic referral template was provided (supplemental eFigure 1A, available with this article at JNCCN.org). Patient referral information included demographics, tumor type, immunotherapy regimen, clinical course, specific referral questions, and request for subspecialist consultation. Members of the IR-tox team discussed cases in real time via a password-protected electronic messaging forum. After 24 hours, a summary of recommendations was sent to referring providers via email, and may have been added to the patient's electronic medical record at the discretion of the referring provider (supplemental eFigure 1B). Further discussion and recommendations beyond 24 hours were used at the discretion of the referring provider and were not included in the 24-hour summary. Members of the IR-tox team met monthly to discuss referrals and identify new clinical, educational, or research needs. Feasibility of the team was defined as receipt of electronic referrals and provision of recommendations within 24 hours of referral. Use of the team was assessed as the proportion of referring providers who used the team's recommendations, collected in a postpilot survey.

\section{Inclusion and Exclusion Criteria, Data Collection}

Patients were included if they were referred to the IR-tox team and were treated at the Sidney Kimmel Comprehensive Cancer Center at Johns Hopkins Hospital (JHH). Included patients had a pathologically confirmed solid tumor or hematologic malignancy and were either receiving, had received, or were due to receive an ICI as standard-of-care or part of a clinical trial. Patients were excluded if they were not treated at JHH and had not received or were not due to start ICIs. Patient, referral, irAE management, and outcome data were collected from electronic referrals, IR-tox team meetings, or extracted from the electronic medical record and collected in an IRB-approved database (IRB00144013). Patients were deemed to have confirmed irAEs if competing diagnoses were excluded, multidisciplinary consensus was reached, and they had either pathologic evidence of irAE or showed clinical improvement with corticosteroid/ irAE-based management. Confirmed irAEs were graded according to CTCAE, version 5.0. ${ }^{10}$ Patients with no or unconfirmed irAEs included those for whom an alternative diagnosis was confirmed or for whom no multidisciplinary consensus could be reached. A flare of a known autoimmune condition was not coded as an irAE. Patients who developed irAEs involving $\geq 2$ organs/systems were defined as having multisystem irAEs. Patients with prior autoimmune conditions or solid organ/bone marrow transplants were defined as having prior autoimmunity or high-risk conditions. Reason for referral was coded as suspected irAE or suitability for ICI. Referral data also included whether diagnostic or management recommendations were sought by referring providers.

\section{Postpilot Survey}

After the first 8 weeks of the pilot program, an anonymous electronic survey was sent to all members of the cancer center, including oncology providers, nurses, and allied staff, to assess the use and utility of the team, types of questions posed to the team, and future suggestions (supplemental eAppendix 1). Providers were asked about their area of practice in oncology, ICI prescribing patterns, and experience in managing irAEs. Providers were unaware they would be surveyed before the postpilot survey was administered.

\section{Statistical Analysis}

Patient, tumor, and treatment characteristics; referral data; and the spectrum of suspected and confirmed irAEs were summarized in frequency and percentage. Patient 
characteristics were compared by irAE grade using the Wilcoxon rank-sum test for continuous variables and Pearson chi-square test for categorical variables. Univariate and multivariate logistic regression were used to obtain odds ratios (ORs) for the effects of treatment, sex, smoking status, age, prior autoimmunity/high-risk conditions, multisystem irAEs, and ICI dose on development of selected irAEs. Clinical factors associated with increased referral $(>1 \mathrm{referral} /$ patient) were explored. Statistical analyses were performed using STATA 14.1 (StataCorp LP). Statistical tests were 2 -sided with a $P$ value $<.05$ considered to indicate statistical significance.

\section{Results}

\section{Feasibility of the IR-Tox Team}

Data were collected from August 1, 2017, through March 30, 2018. The IR-tox team received 122 referrals concerning 107 patients over an 8-month period. Five patients were excluded (did not receive ICIs, $n=4$; not managed at JHH, $\mathrm{n}=1$ ). A total of 117 referrals concerning 102 patients were included in the analysis. For all referrals, the IR-tox team responded with recommendations within 24 hours.
Patient Demographics and Oncologic History CONSORT diagrams of referrals and individual patients are provided in Figure 1 and supplemental eFigure 2, respectively. Indications for referrals included suspected irAE $(n=106 ; 91 \%)$ and suitability for ICIs $(n=11$; $10 \%)$. Of 106 referrals for suspected irAEs, these included 118 potential irAEs. Of the 11 patients referred for suitability for ICIs, 10 (91\%) were for pre-immunotherapy assessment and 1 (9\%) for ICI rechallenge. Of the 10 preimmunotherapy referrals, 7 had prior autoimmune conditions (rheumatoid arthritis, $n=3$; inflammatory bowel disease, $\mathrm{n}=1$; immune thrombocytopenia purpura/Evans syndrome, $\mathrm{n}=1$; myasthenia gravis, $\mathrm{n}=1$; Sjögren syndrome, $n=1$ ), 1 had a prior high-grade irAE (hepatitis), and 2 had other clinical conditions wherein suitability for ICI was questioned (live vaccination, $\mathrm{n}=1$; splenectomy/ increased infection risk, $n=1$ ). Most patients were referred to the team once $(n=87 / 102 ; 85 \%)$ and some were referred twice $(n=15 / 102 ; 15 \%)$.

Patient, treatment, and tumor features of referred patients are summarized in Table 1 . The median age was 64 years (range, $21-91$ years), 54\% of patients were male $(\mathrm{n}=55)$, and $13 \%$ had prior autoimmunity/high-risk conditions $(n=13 ; 9$ autoimmune conditions, 1 solid

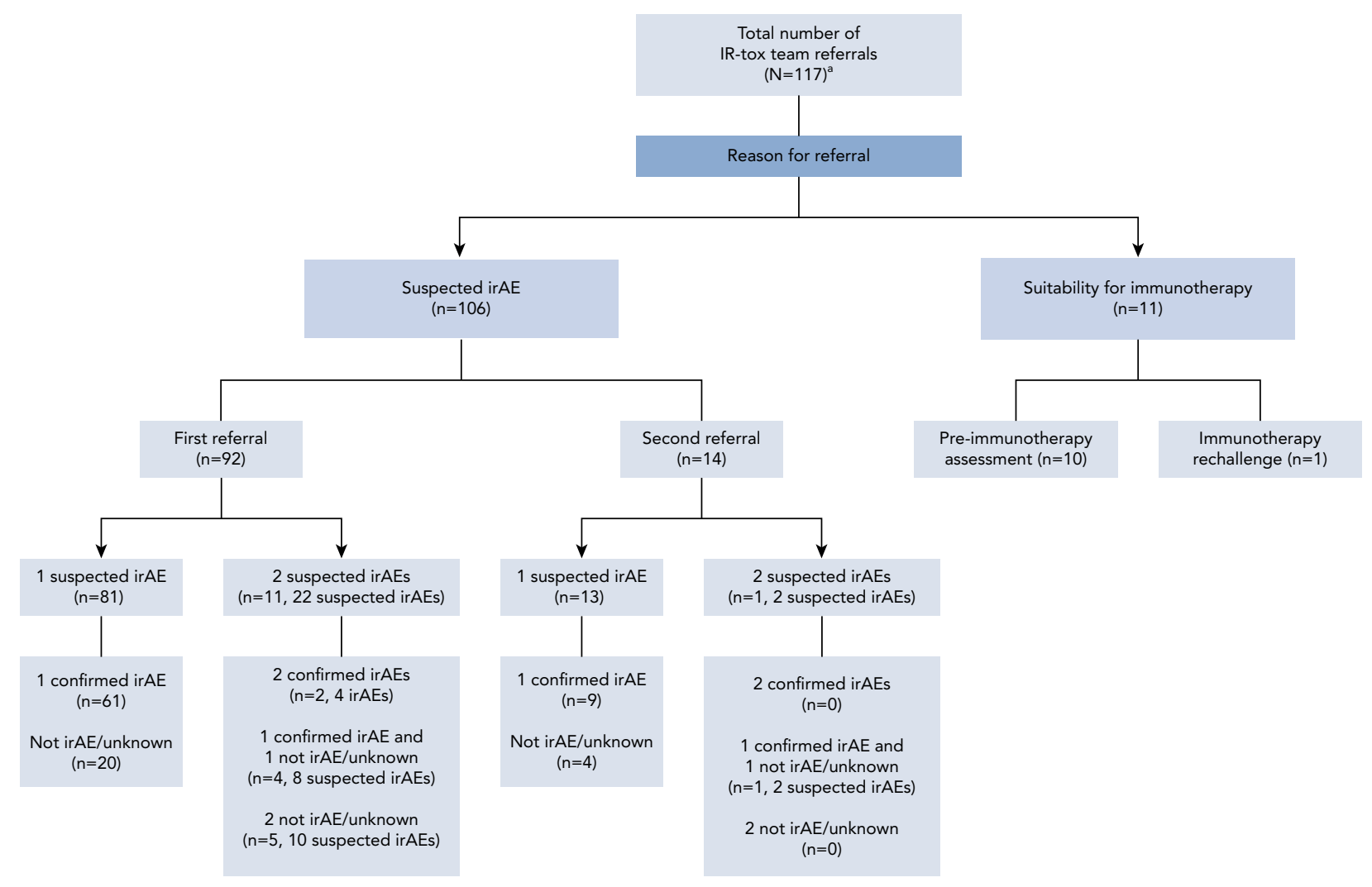

Figure 1. CONSORT diagram of referrals received by the IR-tox team.

Abbreviations: IR-tox, immune-related toxicity; irAE, immune-related adverse event.

aThere were 118 total potential irAEs and 79 confirmed irAEs. 


\begin{tabular}{|c|c|}
\hline Patient Characteristic & n (\%) \\
\hline Median age (range), y & $64(21-91)$ \\
\hline \multicolumn{2}{|l|}{ Sex } \\
\hline Male & $55(53.9)$ \\
\hline Female & $47(46.1)$ \\
\hline \multicolumn{2}{|l|}{ Smoking status } \\
\hline Former/Current & 39 (38.6) \\
\hline Never & $63(61.8)$ \\
\hline \multicolumn{2}{|l|}{ Race } \\
\hline White & $83(81.4)$ \\
\hline Black/African American & $11(10.8)$ \\
\hline Asian & $7(6.9)$ \\
\hline Other & $1(1.0)$ \\
\hline \multicolumn{2}{|l|}{ Prior autoimmunity/high-risk conditions ${ }^{\mathrm{a}}$} \\
\hline Yes & $13(12.7)$ \\
\hline No & $89(87.3)$ \\
\hline \multicolumn{2}{|l|}{ Malignancy type } \\
\hline Thoracic & $34(33.3)$ \\
\hline Gastrointestinal & $18(17.6)$ \\
\hline Melanoma/Skin & $17(16.7)$ \\
\hline Genitourinary & $7(6.9)$ \\
\hline Gynecologic & $8(7.8)$ \\
\hline Head and neck & $6(5.9)$ \\
\hline Breast & $4(3.9)$ \\
\hline Hematologic & $7(6.9)$ \\
\hline Sarcoma & $1(1.0)$ \\
\hline \multicolumn{2}{|l|}{ Treatment setting } \\
\hline Inpatient & $37(36.3)$ \\
\hline Outpatient & $65(63.7)$ \\
\hline \multicolumn{2}{|l|}{ Immune checkpoint inhibitor therapy } \\
\hline Monotherapy & $55(53.9)$ \\
\hline \multicolumn{2}{|l|}{ Combination therapy } \\
\hline + Other immunotherapy & $34(33.3)$ \\
\hline + Chemotherapy & $5(4.9)$ \\
\hline+ Targeted therapy & $4(3.9)$ \\
\hline+ Other agent & $4(3.9)$ \\
\hline Median number of doses at referral (range) & $3(0-40)$ \\
\hline \multicolumn{2}{|l|}{ Receipt of therapy } \\
\hline Standard of care & $50(49.0)$ \\
\hline Clinical trial & $52(51.0)$ \\
\hline
\end{tabular}

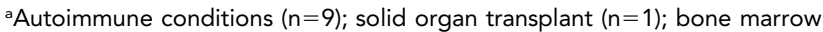
transplant $(n=2)$; prior immune-related adverse event $(n=1)$.

organ transplant, 2 bone marrow transplant, 1 prior irAE). The most common malignancies in referred patients were thoracic $(n=34 ; 33 \%)$, gastrointestinal $(n=18 ; 18 \%)$, and melanoma/skin malignancies $(\mathrm{n}=17 ; 17 \%)$. Patients received either ICI monotherapy $(n=55 ; 54 \%)$ or combinations $(\mathrm{n}=47 ; 46 \%)$, either as standard-of-care $(\mathrm{n}=49$; $49 \%)$ or in clinical trials $(n=52 ; 51 \%)$. Most referrals were received from the outpatient $(n=65 ; 63 \%)$ rather than the inpatient setting $(n=37 ; 36 \%)$. The median number of ICI doses received before referral was 3 (range, $0-40$ ).

\section{Spectrum of irAEs Referred to the IR-Tox Team}

The spectrum of suspected and confirmed irAEs by organ system and individual event is depicted in Figure 2. Of 118 suspected irAEs, 79 irAEs from 68 patients were confirmed by the team (CTCAE grade 1: 13 [16\%]; grade 2: 34 [43\%]; grade $\geq 3: 32[41 \%]$ ). The most frequent irAE referral was for suspected pneumonitis $(n=28 ; 24 \%)$. However, $36 \%$ of suspected pneumonitis referrals were deemed not irAEs (progressive non-small cell lung cancer, $\mathrm{n}=5$; pulmonary infection, $\mathrm{n}=4$; myasthenia gravis, $n=1$ ). This occurred in other suspected irAEs, wherein subsequent imaging, laboratory studies, or clinical features determined that a referral was not for an irAE or consensus was not achieved and attribution was coded as unknown. Aside from pulmonary medicine, referrals were received most frequently for rheumatology $(n=21 ; 18 \%)$, gastroenterology $(n=15 ; 13 \%)$, dermatology $(n=15 ; 13 \%)$, endocrinology $(n=14 ; 13 \%)$, and neurology $(\mathrm{n}=10 ; 8 \%)$.

The most common confirmed irAEs included pneumonitis $(n=18 ; 23 \%)$, arthritis $(n=13 ; 16 \%)$, dermatitis $(\mathrm{n}=12 ; 15 \%)$, and diarrhea/colitis $(\mathrm{n}=8 ; 10 \%)$ (Figure 2B). One new case of osteitis was identified that had not previously been reported in the literature. A total of 58 patients (85\%) had irAEs involving 1 organ/system, whereas 10 patients (15\%) had multisystem irAEs. The spectrum of irAEs stratified by treatment type, organ system, and grade is depicted in Figure 3. In multivariate analyses, receipt of combination ICIs $(n=34)$ was associated with an increased risk of grade $\geq 3$ irAEs (OR, 6.5; 95\% CI, 1.70-24.6; $P=.018$ ) and colitis (OR, 12.9; $95 \%$ CI, $1.2-143.7 ; P=.037)$ and a trend toward multisystem irAEs (OR, 3.0; 95\% CI, 0.9-9.3; $P=.06$ ). Patients with prior autoimmunity/high-risk conditions $(n=13)$ were more likely to develop pneumonitis (OR, 27.2; 95\% CI, 1.9-385.9; $P=.015$ ), and trended toward a higher risk for grade $\geq 3$ irAEs (OR, 8.9; 95\% CI, 0.8-95.8; $P=.07$ ). Importantly, an increased risk for multiple referrals $(n=13)$ to the IR-tox team was seen in patients with multisystem irAEs (OR, 8.1; 95\% CI, 1.9-34.8; $P=.005)$ and/or those receiving combination ICIs (OR, 6.0; 95\% CI, 1.1-32.6; $P=.035$ ).

\section{Recommendations by the IR-Tox Team}

IR-tox team referral data and recommendations are depicted in Table 2. Referrals were received from 
A

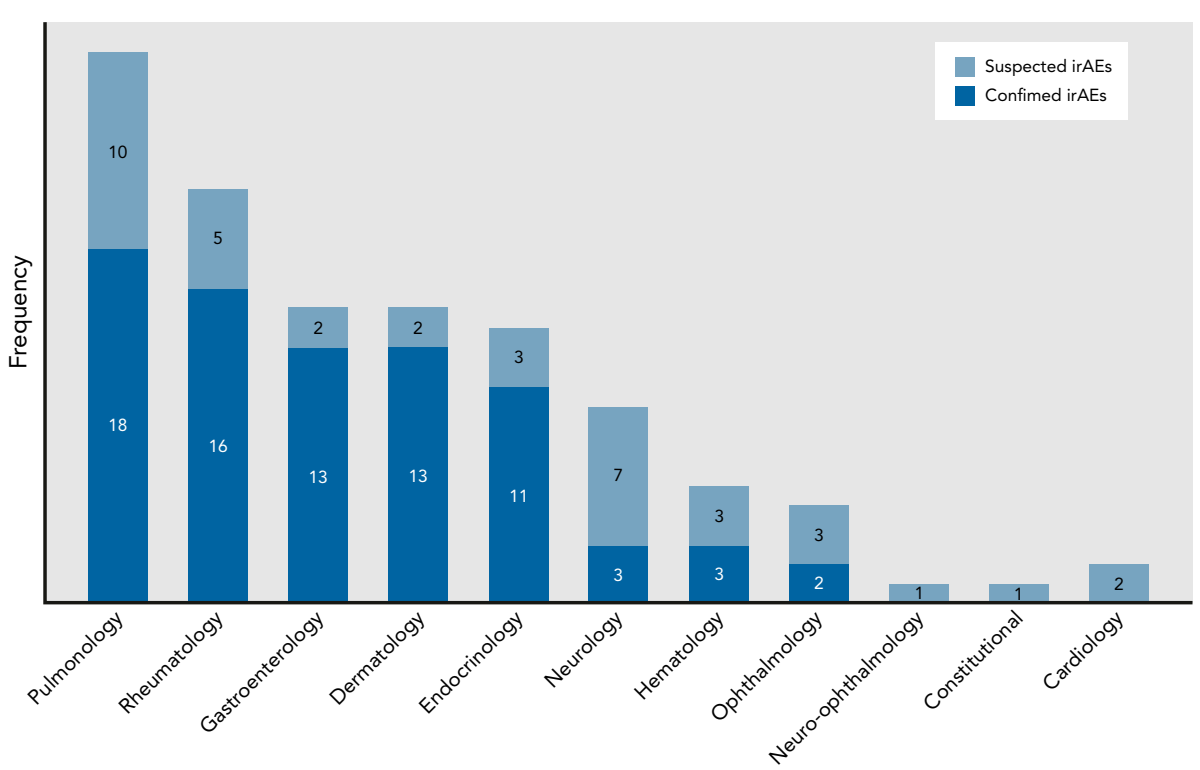

B

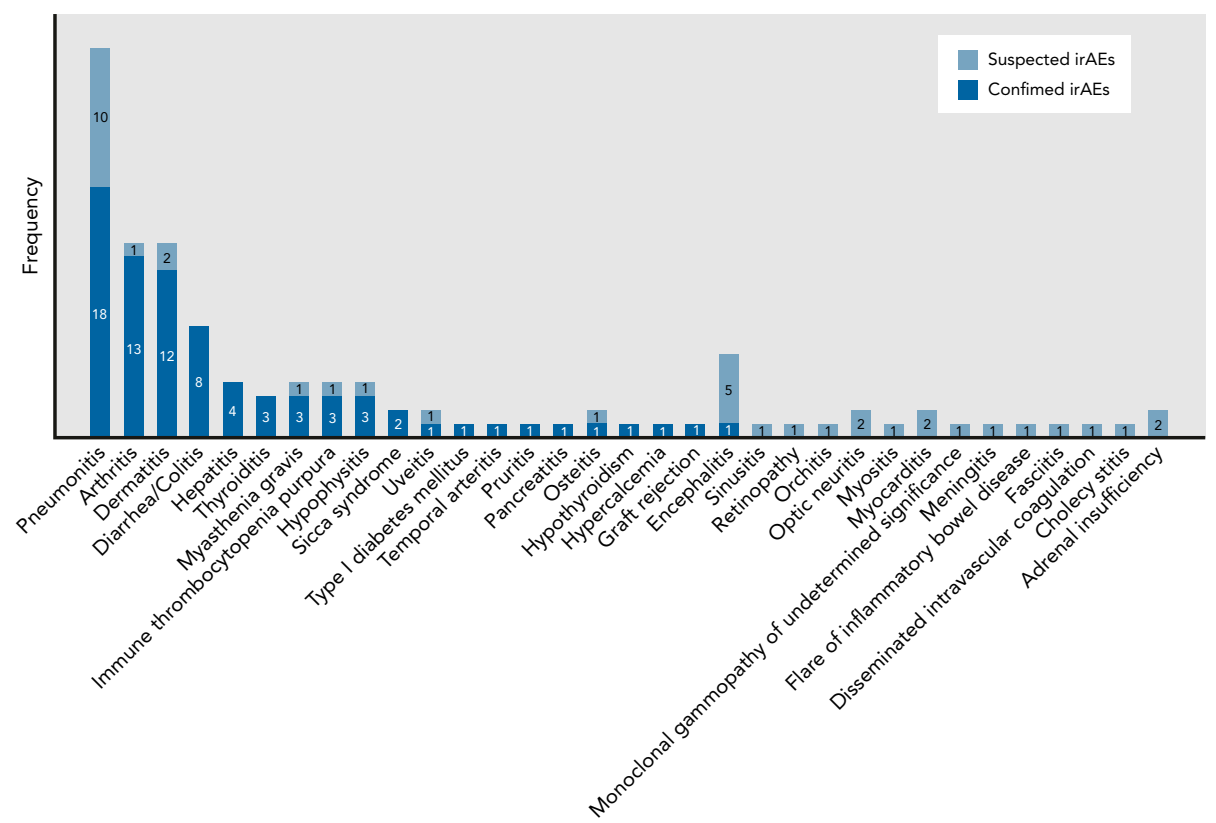

Figure 2. Spectrum of irAE referrals by (A) frequency of suspected and confirmed irAE by organ system and (B) specific suspected and confirmed irAE.

Abbreviation: irAE, immune-related adverse event.

attending physicians $(n=81 ; 69 \%)$, fellows $(n=12 ; 10 \%)$, and nurses $(n=24 ; 21 \%)$. In patients being treated as part of clinical trials, specific irAE management recommendations outlined in the study protocol were adhered to. Among referrals received regarding suitability for ICIs, $45 \%$ of referring providers $(n=5 / 11)$ sought management recommendations. For the 118 suspected irAEs, the IR-tox team was mostly asked for diagnostic recommendations $(n=110 ; 93 \%)$; in $95 \%$ $(n=75 / 79)$ of confirmed irAEs, the team was asked for management recommendations.

In the 17 patients with no/unknown irAEs in whom a management recommendation was sought, the team advised supportive care only in $94 \%(n=16)$ and immunosuppression in 1 patient with a flare of known inflammatory bowel disease. Subspecialist consultations were obtained for referred patients regardless of whether they had a confirmed irAE, with $51 \%$ of 
A

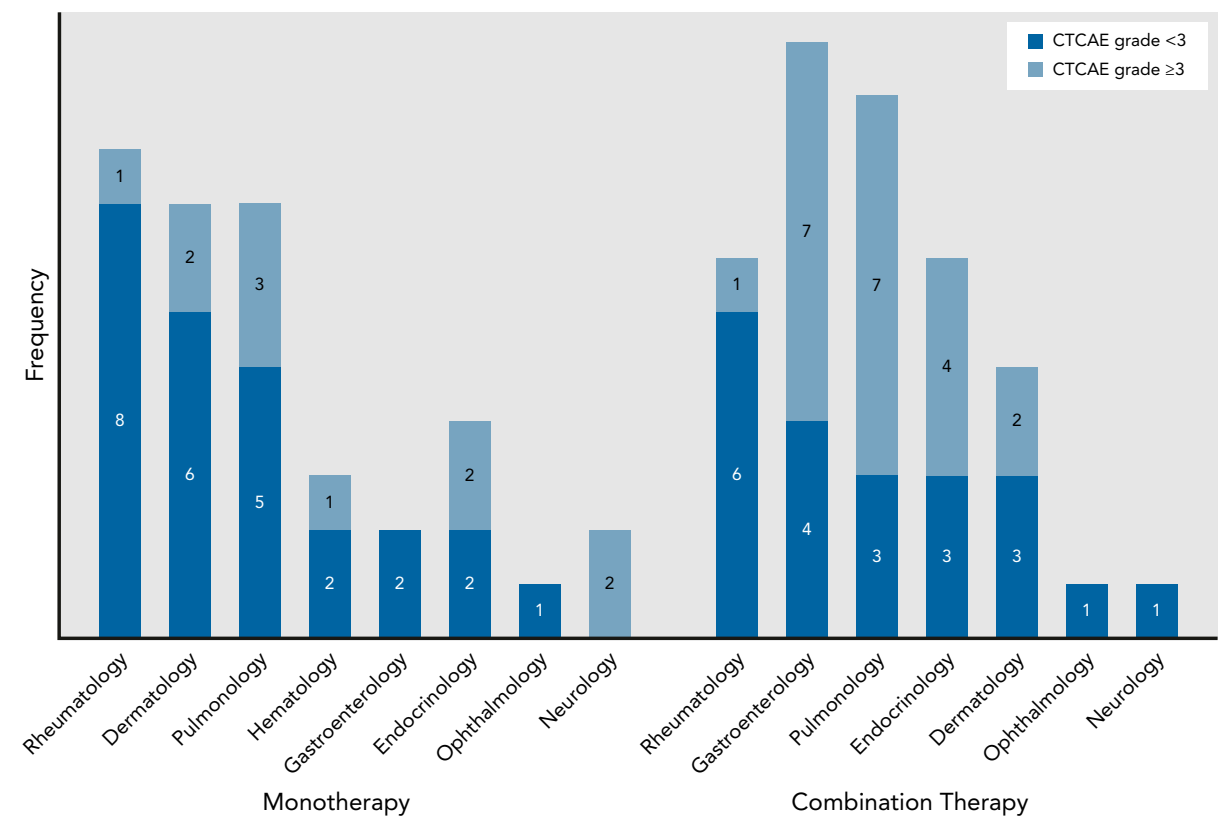

B

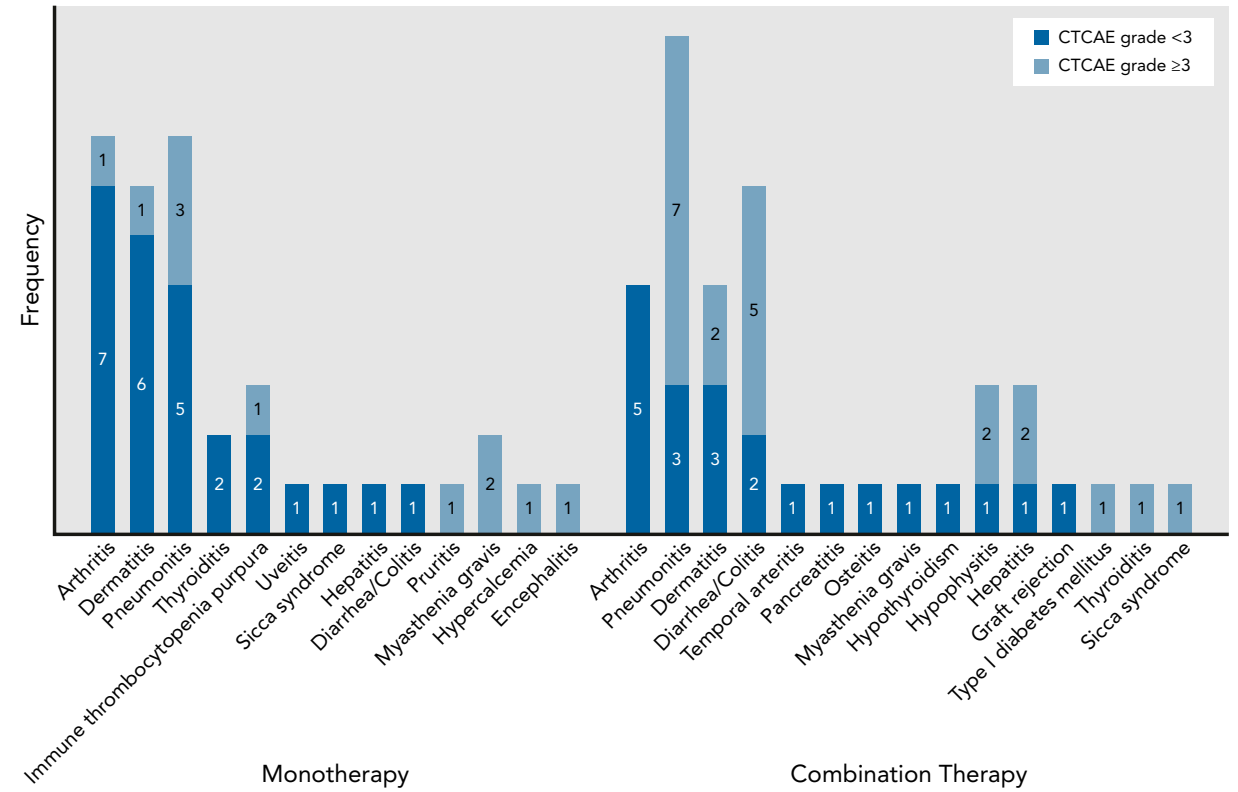

Figure 3. irAEs confirmed by IR-tox team by (A) grade and organ system and (B) grade and specific irAE. Abbreviations: irAE, immune-related adverse event; IR-tox, immune-related toxicity.

non-irAEs/unknown and 35\% of confirmed irAEs requiring more than one medicine consultation.

In all confirmed irAEs, invasive diagnostic testing was more likely to be undertaken in high-grade versus low-grade irAEs (59\% vs $37 \% ; P=.006)$. In addition, $39 \%$ of patients $(n=31)$ with high-grade irAEs were managed with corticosteroids \pm additional immunosuppression, and significant differences in management recommendations were seen in low- versus high-grade irAEs $(P<.001)$.

\section{Outcomes of Confirmed irAEs}

Clinical outcomes of confirmed irAEs referred to the IRtox team are summarized in supplemental eAppendix 2 . All low-grade irAEs completely resolved/improved (39/47; 83\%) or stabilized (17\%; 8/47), whereas $13 \%$ of 
Table 2. IR-Tox Team Referral Data and Recommendations

\begin{tabular}{|c|c|c|c|}
\hline \multirow[b]{2}{*}{ Reason for Referral } & \multicolumn{2}{|c|}{ Suspected irAE $(N=118)$} & \multirow[b]{2}{*}{$\begin{array}{l}\text { Suitability for Immunotherapy, } n \text { (\%) } \\
\qquad(\mathbf{N}=11)\end{array}$} \\
\hline & $\begin{array}{l}\text { Not irAE/Unknown, } n(\%) \\
(\mathbf{N}=39)\end{array}$ & $\begin{array}{l}\text { Confirmed irAE, n (\%) } \\
(\mathrm{N}=79)\end{array}$ & \\
\hline \multicolumn{4}{|l|}{ Question for the IR-tox team } \\
\hline \multicolumn{4}{|l|}{ Suitability for immunotherapy } \\
\hline Yes & $0(0)$ & $0(0)$ & $11(100)$ \\
\hline No & $39(100)$ & $79(100)$ & $0(0)$ \\
\hline \multicolumn{4}{|l|}{ Diagnostic } \\
\hline Yes & $38(97)$ & $72(91)$ & 1 (9) \\
\hline No & $1(3)$ & $7(9)$ & $10(91)$ \\
\hline \multicolumn{4}{|l|}{ Management } \\
\hline Yes & $17(44)$ & 75 (95) & $5(45)$ \\
\hline No & $22(56)$ & $4(5)$ & $6(55)$ \\
\hline \multicolumn{4}{|l|}{ IR-tox team recommendations } \\
\hline Total diagnostic recommendations & 38 & 72 & 1 \\
\hline No further diagnostic tests required & $6(15)$ & $0(0)$ & $0(0)$ \\
\hline Noninvasive diagnostic tests & $24(62)$ & $44(56)$ & $1(9)$ \\
\hline Invasive diagnostic test & $8(20)$ & $28(35)$ & $0(0)$ \\
\hline Total management recommendations & 17 & 75 & 5 \\
\hline Supportive care only & $16(41)$ & $14(18)$ & $2(18)$ \\
\hline Corticosteroids only & $0(0)$ & $46(58)$ & $1(9)$ \\
\hline Additional immunosuppression only & $0(0)$ & $4(5)$ & $2(18)$ \\
\hline Steroids + additional immunosuppression & $1(3)$ & $11(14)$ & $0(0)$ \\
\hline \multicolumn{4}{|l|}{ IR-tox team general recommendations } \\
\hline \multicolumn{4}{|l|}{ Medicine consultation } \\
\hline One & $12(31)$ & $47(59)$ & $4(36)$ \\
\hline Multiple & $20(51)$ & $28(35)$ & $1(9)$ \\
\hline None & $6(8)$ & $4(6)$ & $6(55)$ \\
\hline
\end{tabular}

Abbreviations: irAE, immune-related adverse event; IR-tox, immune-related toxicity.

high-grade irAEs (4/32) worsened. Of the 4 patients whose irAEs worsened, 3 died of their irAEs (nephritis: 1 ; pneumonitis: 1; encephalitis: 1).

\section{Use of the IR-Tox Team: Postpilot Survey by Users} Among 57 respondents to the postpilot survey at JHH (physician, $\mathrm{n}=35$; nurse practitioner/physician's assistant, $n=7$; registered nurse, $n=15$; other, $n=4$ ), the frequency of irAE evaluation varied: $3.6 \%$ evaluated them daily, $8.9 \%$ two to three times per week, $25 \%$ once per week, and $51.8 \%$ once per month. A total of 34 respondents used the IR-tox team service $(59.7 \%)$, whereas 23 did not $(40.3 \%)$. Of those who contacted the team, $100 \%$ of providers used all $(n=19 ; 55.9 \%)$ or some $(n=15 ; 44.1 \%)$ of the recommendations. Importantly, $73.5 \%(\mathrm{n}=25)$ of referring providers said the IR-tox team recommendations changed their diagnostic evaluation or management of immune-related toxicity. Overall, most respondents who used the IR-tox team rated it as definitely or probably helpful $(n=33 ; 97 \%)$ (supplemental eFigure 3); 39 respondents $(72.2 \%)$ thought a regular irAE tumor board-style meeting, in addition to the electronic referral system, would definitely or probably be beneficial.

\section{Discussion}

This is the first report of a multidisciplinary team for immune-related toxicity. We demonstrated the feasibility of an electronic referral system for patients with irAEs and related issues to multiple subspecialists. We also demonstrated use of the IR-tox team, because all surveyed providers who contacted the team used all or some of the recommendations, and most changed their management based on team recommendations. These data highlight important functions an IR-tox team 
can provide in guiding clinical care and disseminating knowledge to providers less familiar with irAEs, and standardizing irAE management across an institution. We also identified toxicities that require subspecialist input, of which pneumonitis, arthritis, and dermatitis were the most common. Results of our program highlight patient populations more likely to use this service, such as those receiving combination ICIs and those with multisystem irAEs. With expanding use of combination ICIs in multiple tumor types, ${ }^{11-14}$ the absolute number of patients treated with these regimens is likely to increase, supporting the need for an IR-tox team.

There are several unique features of the IR-tox team program. The multidisciplinary nature of the group facilitated identification of newly recognized irAEs and multisystem events. Similarly, the CAR-T-cell-therapyassociated TOXicity (CARTOX) Working Group leverages oncologists and subspecialists across institutions ${ }^{15}$ to guide management of CAR T-cell toxicities. We envisage that IR-tox teams across institutions and countries, treating diverse populations with ICIs, may collaborate to guide research and refine current irAE guidelines.

The prospective nature of irAE data collection through the IR-tox team created an opportunity to identify alternative diagnoses for suspected irAEs. For example, patients not deemed to have pneumonitis by the team had progressive cancer or respiratory infection, and in one case a different irAE (myasthenia gravis). Likewise, only $30 \%$ of suspected neurologic irAE referrals were found to be irAEs. In contrast, all patients with suspected colitis had this diagnosis confirmed. This emphasizes the diagnostic dilemma that exists for pneumonitis and neurologic irAEs in particular.

Another unique feature of the IR-tox team program was its electronic platform to engage multiple specialists. This format reduces administrative burden and could be leveraged for telemedicine, to provide irAE expertise to patients being managed in limited-access settings. ${ }^{16} \mathrm{Al}-$ though other immune-related toxicity initiatives have been proposed as part of inpatient oncology services, this virtual team allowed for specialist input for outpatients, which may be more applicable. Lastly, more than half of IR-tox team referrals were for patients enrolled in clinical trials, to ascertain an irAE diagnosis, or for management advice when specific guidance was not outlined in the study protocol. This was especially relevant for rare toxicities that may not have been addressed individually in study protocols. Attribution of toxicity to a study drug has important implications for cancer drug development, and may identify a unique and important use for a multidisciplinary team. Electronic or in-person IR-tox teams could serve as independent adjudicators of
irAE attributions, and resolve relative discrepancies in irAE attribution between study sites, noted in irAE publications. ${ }^{17}$

One limitation of this study was the lack of a control group (ie, patients for which the IR-tox team was not available for consultation). Future studies might aim to evaluate time to referral, time to irAE treatment, or time to irAE resolution in patients referred to the IR-tox team versus not. During the time period of our study, there were likely patients who were directly referred to medicine subspecialists and therefore not captured in this dataset. These patients may have had lower grade or less complex irAEs. Additionally, known areas of interest for members of the IR-tox team may have influenced referral patterns (eg, pneumonitis and arthritis are areas of interest of the co-chairs). Although we were able to bring together a large team of irAE subspecialists at our institution, this may be more challenging to re-create outside of academic medicine. In addition, although an electronic platform allowed access to a variety of specialists without an unacceptable wait time, some clinical scenarios warrant emergent management. We attempted to account for this with an immediate response message, urging providers to direct patients to the emergency room in urgent situations.

\section{Conclusions}

This pilot program demonstrates that an electronic referral system for immune-related toxicities was feasible, used, and informative about patterns of irAEs requiring subspecialist care. These data lay the foundation for the development of local, national, and international IR-tox teams with the goal of optimizing multidisciplinary clinical care, supporting irAE education, leveraging virtual expertise to limited access settings, and applying to clinical trials. Further research in this area is likely to be fostered by a comprehensive irAE database, and future studies aimed at evaluating whether an IR-tox team approach versus ad hoc specialist referral could influence important patient outcomes, such as hospitalization rates, time on corticosteroid treatment, or time to restarting immunotherapy, in patients who develop irAEs.

\section{Acknowledgments}

The authors would like to acknowledge all members of the IRTox team who contributed to the care of the patients included in this manuscript, and those who referred patients to this pilot program. We extend special thanks to the team members who were not included as co-authors but contributed to the development of this manuscript: Jennifer Mammen, MD; Inbal Sander MD; Joanna Melia, MD; Lyle Ostrow, MD, PhD; Meghan Berkenstock, MD; Alan Baer, MD; Lonny Yarmus, DO; Patricia Brothers, BSN; Anna Ferguson, BSN; Maureen Berg, 
BSN; Dung T. Le, MD; Kristen A. Marrone, MD; Leisha A. Emens, MD, PhD; William J. Sharfman, MD; and Elizabeth M. Jaffee, MD, PhD.

Submitted October 3, 2018; accepted for publication January 4, 2019

Author contributions: Study concept and design: Naidoo, Lipson, Forde, Shah, Pardoll, Bingham, Brahmer, Cappelli. Data acquisition: Naidoo, Zhang, Cappelli. Data analysis and interpretation: All authors. Drafting of manuscript: All authors.

Disclosures: Dr. Naidoo has disclosed that she is a consultant for AstraZeneca, Bristol-Myers Squibb, and Takeda; receives grant/research support from Merck, AstraZeneca/Medlmmune, Calithera, and Kyowa Hakko Kirin; and receives honoraria from AstraZeneca/Medlmmune and Bristol-Myers Squibb. Dr. Lipson has disclosed that he is a consultant for Bristol-Myers Squibb, Novartis, EMD Serono, Array BioPharma, Regeneron/Sanofi Genzyme, Macrogenics, Merck, and Millennium; and receives grant/research support from Bristol-Myers Squibb, Merck, and Sysmex. Dr. Forde has disclosed that he is a consultant for Bristol-Myers Squibb, AstraZeneca/Medlmmune, Novartis, AbbVie, Boehringer, Celgene, and Eli Lilly, and receives grant/ research support from Bristol-Myers Squibb, AstraZeneca/Medlmmune Novartis, and Kyowa Hakko Kirin. Dr. Thorne has disclosed that she is a consultant for Gilead, Santen, and AbbVie. Dr. Shanbhag has disclosed that he receives grant/research support from Daiichi-Sankyo, and is a scientific advisor for Takeda Oncology. Ms. Riemer has disclosed that she is a consultant for Bristol-Myers Squibb, EMD Serono, and AstraZeneca/ Medlmmune. Dr. Shah has disclosed that she serves as a consultant to Bristol-Myers Squibb. Dr. Pardoll has disclosed that he is a consultant for Aduro Biotech, Amgen, AstraZeneca/Medlmmune/Amplimmune, Bayer Camden Nexus II, Compugen, DNATRIX, Dynavax Technologies Corporation, Five Prime Therapeutics, Rock Springs Capital, Tizona, Janssen, Immunomic Therapeutics, Merck, WindMIL, FLX Bio, and Ervaxx; receives grant/research support from Bristol-Myers Squibb; and has an IP License from Bristol-Myers Squibb, Compugen, and Potenza. Dr. Bingham has disclosed that he is a consultant for and receives grant/research support from Bristol-Myers Squibb. Dr. Brahmer has disclosed that she is a consultant for Bristol-Myers Squibb, AstraZeneca/Medlmmune, Genentech, Celgene, Eli Lilly, Merck, Amgen, and Syntax, and receives grant/research support from Bristol-Myers Squibb, AstraZeneca/ Medlmmune, Incyte, and Merck. Dr. Cappelli has disclosed that she receives grant/research support from Bristol-Myers Squibb. The remaining authors have not received any financial consideration from any person or organization to support the preparation, analysis, results, or discussion of this article.

Correspondence: Jarushka Naidoo, MBBCh, Johns Hopkins Bayview, 301 Mason Lord Drive, Baltimore, MD 21224. Email: jnaidoo1@jhmi.edu

\section{References}

1. Lee B, Kim K, Choi JY, et al. Efficacy of the multidisciplinary tumor board conference in gynecologic oncology: a prospective study. Medicine (Baltimore) 2017;96:e8089.

2. Wheless SA, McKinney KA, Zanation AM. A prospective study of the clinical impact of a multidisciplinary head and neck tumor board. Otolaryngol Head Neck Surg 2010;143:650-654.

3. van Hagen $P$, Spaander MC, van der Gaast $A$, et al. Impact of a multidisciplinary tumour board meeting for upper-GI malignancies on clinical decision making: a prospective cohort study. Int J Clin Oncol 2013;18:214-219.

4. Kurpad R, Kim W, Rathmell WK, et al. A multidisciplinary approach to the management of urologic malignancies: does it influence diagnostic and treatment decisions? Urol Oncol 2011;29:378-382.

5. Schmidt HM, Roberts JM, Bodnar AM, et al. Thoracic multidisciplinary tumor board routinely impacts therapeutic plans in patients with lung and esophageal cancer: a prospective cohort study. Ann Thorac Surg 2015;99: 1719-1724.

6. van der Velden DL, van Herpen CM, van Laarhoven HW, et al. Molecular tumor boards: current practice and future needs. Ann Oncol 2017;28: 3070-3075.

7. Postow MA, Sidlow R, Hellmann MD. Immune-related adverse events associated with immune checkpoint blockade. N Engl J Med 2018;378:158-168.

8. Gupta A, De Felice KM, Loftus EV Jr, et al. Systematic review: colitis associated with anti-CTLA-4 therapy. Aliment Pharmacol Ther 2015;42: 406-417.

9. Postow MA Managing immune checkpoint-blocking antibody side effects. Am Soc Clin Oncol Educ Book 2015;35:76-83.
10. U.S. Department of Health and Human Services. Common Terminology Criteria for Adverse Events (CTCAE). Version 5.0. Available at: https:// ctep.cancer.gov/protocolDevelopment/electronic_applications/docs/ CTCAE_v5_Quick_Reference_8.5x11.pdf. Accessed August 24, 2018.

11. Wolchok JD, Chiarion-Sileni V, Gonzalez R, et al. Overall survival with combined nivolumab and ipilimumab in advanced melanoma. N Engl J Med 2017;377:1345-1356.

12. Hellmann MD, Ciuleanu TE, Pluzanski A, et al. Nivolumab plus ipilimumab in lung cancer with a high tumor mutational burden. N Engl J Med 2018; 378:2093-2104.

13. Motzer RJ, Tannir NM, McDermott DF, et al. Nivolumab plus ipilimumab versus sunitinib in advanced renal-cell carcinoma. N Engl J Med 2018;378: 1277-1290.

14. Antonia SJ, López-Martin JA, Bendell J, et al. Nivolumab alone and nivolumab plus ipilimumab in recurrent small-cell lung cancer (CheckMate 032): a multicentre, open-label, phase 1/2 trial. Lancet Oncol 2016;17: 883-895.

15. Neelapu SS, Tummala S, Kebriaei P, et al. Chimeric antigen receptor T-cell therapy-assessment and management of toxicities. Nat Rev Clin Oncol 2018;15:47-62.

16. Zhou C, Crawford A, Serhal E, et al. The impact of Project ECHO on participant and patient outcomes: a systematic review. Acad Med 2016 91:1439-1461.

17. Lisberg A, Tucker DA, Goldman JW, et al. Treatment-related adverse events predict improved clinical outcome in NSCLC patients on KEYNOTE-001 at a single center. Cancer Immunol Res 2018;6:288-294.

See JNCCN.org for supplemental online content. 
Supplemental online content for:

\section{A Multidisciplinary Toxicity Team for Cancer Immunotherapy-Related Adverse Events}

Jarushka Naidoo, MBBCh; Jiajia Zhang, MD, PhD; Evan J. Lipson, MD; Patrick M. Forde, MBBCh; Karthik Suresh, MD; Kendall F. Moseley, MD; Seema Mehta, MD; Shawn G. Kwatra, MD; Alyssa M. Parian, MD; Amy K. Kim, MD; John C. Probasco, MD, PhD; Rosanne Rouf, MD; Jennifer E. Thorne, MD, PhD; Satish Shanbhag, MD; Joanne Riemer, RN, BSN; Ami A. Shah, MD; Drew M. Pardoll, MD, PhD; Clifton O. Bingham III, MD; Julie R. Brahmer, MD, MHS; and Laura C. Cappelli, MD, MHS

J Natl Compr Canc Netw 2019;17(6):712-720

eFigure 1: Representative Templates to and From IR-Tox Team eFigure 2: CONSORT Diagram

eFigure 3: Selected Results of the Electronic Postpilot Survey eAppendix 1: Postpilot Questionnaire About the IR-Tox Team eAppendix 2: Clinical Outcomes of Confirmed irAEs 


\begin{tabular}{|l|l|}
\hline A & B \\
Referral to IR-Tox Team & IR-Tox Team Email Recommendation Template \\
\hline Patient initials: & Recommendation: \\
Medical record number: & - Summary of recommendations \\
Referring provider: & Request for medicine referral: \\
Primary provider: & - Name of provider \\
Name of immunotherapy agent(s): & - Provider cc'ed \\
Duration of immunotherapy: $x$ months (y doses) & Disclaimer: Recommendations from the IR-tox team will \\
Tumor type: & be provided within 24 hours of referral. The IR-tox team \\
Diagnostic evaluation ordered/completed: & provides recommendations; however, decisions regarding \\
Brief summary: & the diagnostic evaluation and management of referred \\
Specific question: & patients will be made by the patient's primary provider. \\
Request for medicine referral: & \\
\hline
\end{tabular}

eFigure 1. Representative templates of information included for (A) referral to IR-tox team and (B) recommendations provided by IR-tox team 24 hours after referral.

Abbreviation: IR-tox, immune-related toxicity.

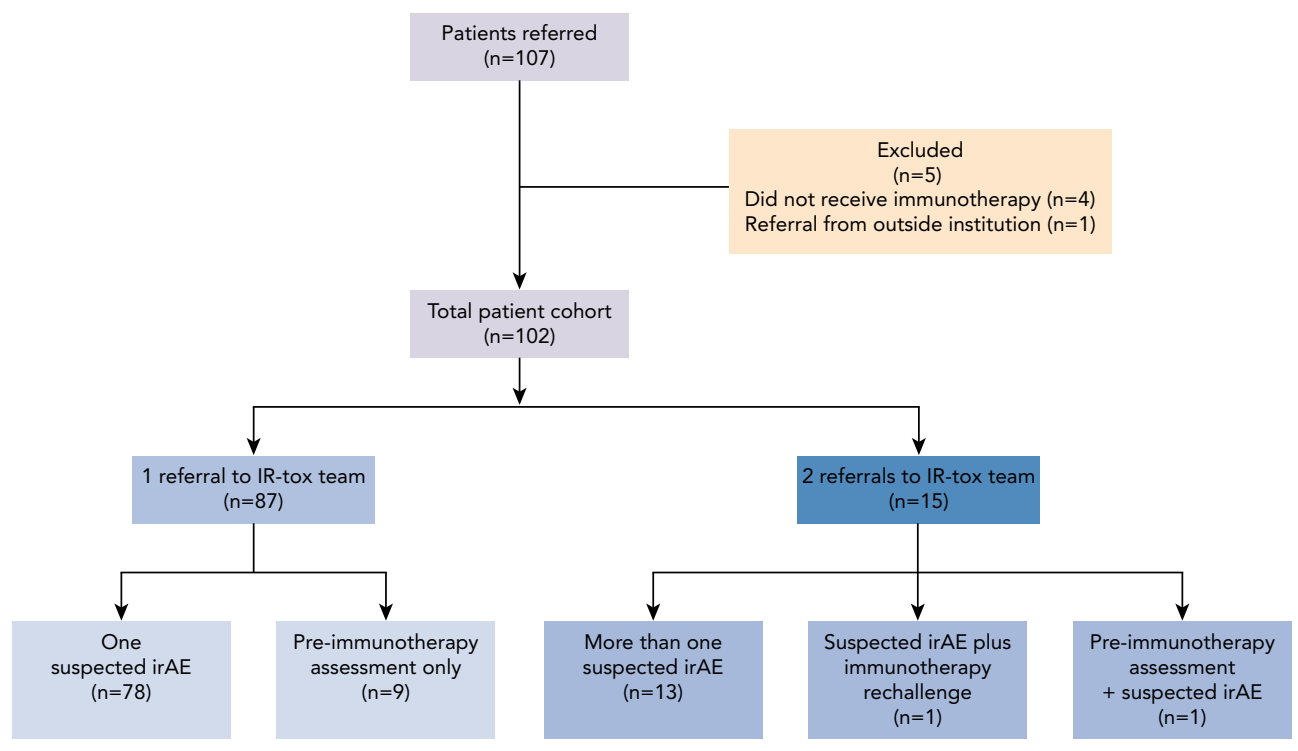

eFigure 2. CONSORT diagram of patients referred to the IR-tox team.

Abbreviations: IR-tox, immune-related toxicity; irAE, immune-related adverse event. 


\section{2 - Naidoo et al}

IR-Tox Team Use

Did you find the IR-tox team service to be helpful?

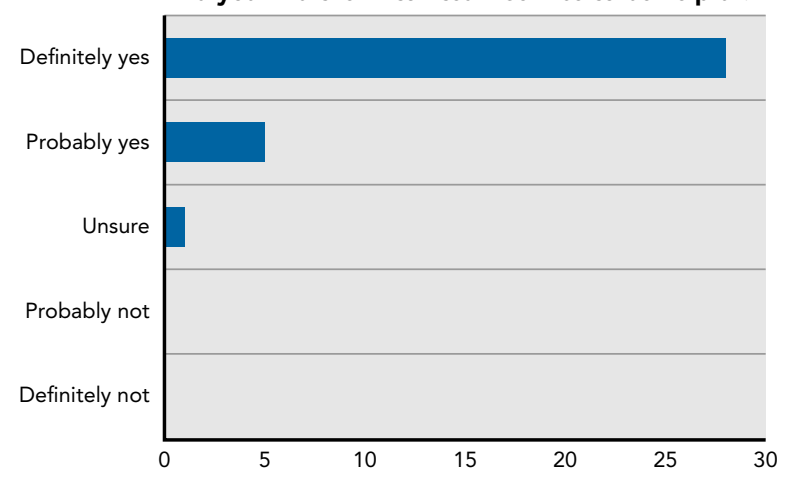

What topic(s) did you ask the IR-tox team about?

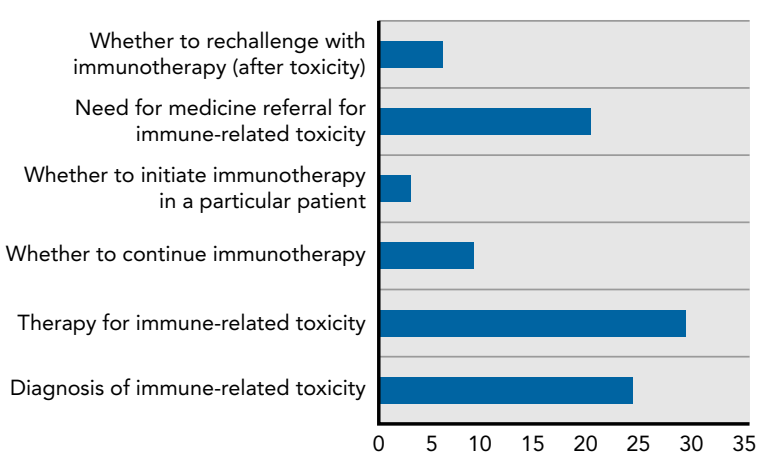

Future Uses for the IR-Tox Team

Would you support an immune-related toxicity registry/enrollment of patients?

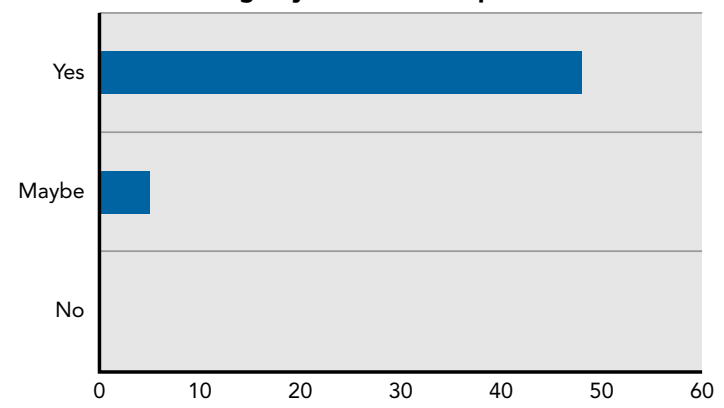

What other resources could the IR-tox team provide?

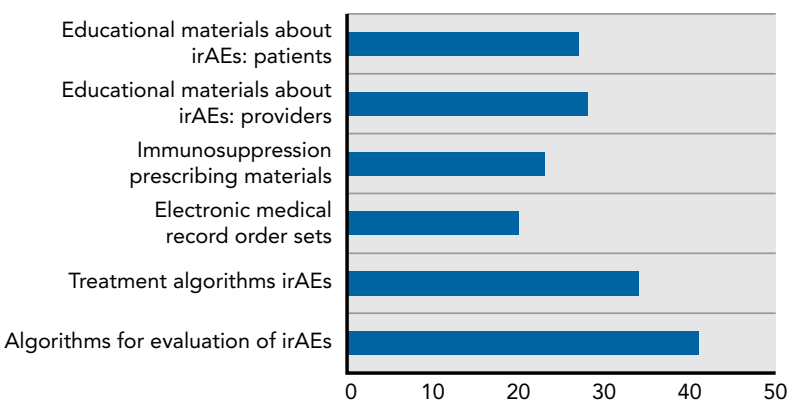

eFigure 3. Selected results of the electronic postpilot survey sent to oncology providers in the cancer center, after the IR-tox team pilot program. Abbreviations: IR-tox, immune-related toxicity; irAE, immune-related adverse event. 


\section{eAppendix 1. Postpilot Questionnaire About the IR-Tox Team}

\begin{tabular}{|c|c|}
\hline Q1 & What area of oncology do you primarily work in? \\
\hline Q2 & What type of provider are you? \\
\hline Q3 & How often do you prescribe immunotherapy for cancer treatment/evaluate patients on immunotherapy? \\
\hline Q4 & What kind of immunotherapy do you prescribe? (check all that apply) \\
\hline Q5 & How often do you encounter suspected immune-related toxicity? \\
\hline Q6 & How often do you encounter immune-related toxicity that requires an intervention? \\
\hline Q7 & Have you contacted the IR-tox team? \\
\hline Q8 & What topic(s) did you ask the team about? (check all that apply) \\
\hline Q9 & Did you use the specific suggestions provided by the IR-tox team? \\
\hline Q10 & Did the IR-tox team recommendations change your approach to evaluation or management of immune-related toxicity? \\
\hline Q11 & Did you find the IR-tox team service to be helpful? \\
\hline Q12 & What format would you prefer to contact the IR-tox team? (choose one) \\
\hline Q13 & What other resources could the IR-tox team provide that would be helpful? (check all that apply) \\
\hline Q14 & How would you prefer to access information on IR-tox? \\
\hline Q15 & Do you think there would be a benefit to a regular tumor board-style meeting about IR-tox? \\
\hline Q16 & How often should a tumor board-style meeting for IR-tox take place? \\
\hline Q17 & Would you support an immune-related toxicity registry/enrollment of patients with irAEs for research purposes? \\
\hline Q18 & Do you want a separate irAEs outpatient clinic? \\
\hline Q19 & Do you want a separate irAEs urgent care? \\
\hline Q20 & Would you attend an irAEs masterclass if offered? \\
\hline Q21 & Please provide any additional feedback \\
\hline
\end{tabular}

Abbreviations: IR-tox, immune-related toxicity; irAE, immune-related adverse event.

\begin{tabular}{|c|c|c|}
\hline \multicolumn{3}{|c|}{$\begin{array}{l}\text { eAppendix 2. Clinical Outcomes of Confirmed } \\
\text { irAEs }\end{array}$} \\
\hline Clinical Outcomes & $\begin{array}{c}\text { Grade } 1-2 \\
(n=47 ; 60 \%) \\
n(\%)\end{array}$ & $\begin{array}{c}\text { Grade } \geq 3 \\
(n=32 ; 40 \%) \\
n(\%)\end{array}$ \\
\hline \multicolumn{3}{|l|}{ irAE1 ${ }^{a}$} \\
\hline Completely resolved & $2(4)$ & $3(9)$ \\
\hline Improved & $32(68)$ & $20(63)$ \\
\hline Stabilized & $6(13)$ & $3(9)$ \\
\hline Worsened & $0(0)$ & $3(9)$ \\
\hline \multicolumn{3}{|l|}{ irAE2 ${ }^{b}$} \\
\hline Completely resolved & $3(7)$ & $0(0)$ \\
\hline Improved & $2(4)$ & $1(3)$ \\
\hline Stabilized & $2(4)$ & $1(3)$ \\
\hline Worsened & $0(0)$ & $1(3)$ \\
\hline
\end{tabular}

Abbreviation: irAE, immune-related adverse event.

aFirst irAE prompting referral.

becond irAE prompting referral. 